\title{
A Special Treatment of Vulvar Cancer with Pelvic Organ Prolapse: A Case Report
}

qin tang ( $\square$ 1226142591@qq.com)

Sichuan University WCSUH: Sichuan University West China Second University Hospital ping wang ( $\nabla$ qintangljs@126.com )

Sichuan University WCSUH: Sichuan University West China Second University Hospital

\section{Case report}

Keywords: female genital neoplasms, urogenital cancer, uterine disease, urogenital prolapse, vaginal prolapse, vaginal vault prolapse

Posted Date: March 18th, 2021

DOI: https://doi.org/10.21203/rs.3.rs-334285/v1

License: (1) This work is licensed under a Creative Commons Attribution 4.0 International License. Read Full License 


\section{Abstract}

Background: Pelvic organ prolapse is a common benign condition in women, defined as the descent of one or more of the following: anterior vaginal wall; posterior vaginal wall; uterus (cervix); and apex of the vagina (vaginal vault or cuff scar after hysterectomy). Vulvar cancer is uncommon, accounting for $2-5 \%$ of gynecologic malignancies. Vulvar cancer and pelvic organ prolapse tend to occur in elderly individuals with other complications, such as heart disease, hypertension and diabetes. Risk during the perioperative period is very high; therefore, gynecologists should provide the most appropriate and comprehensive treatment plan to achieve a good prognosis.

Case presentation: The patient's main concerns is vulva pruritus and vulva mass prolapse. Important clinical examinations include vulvar biopsy suggesting vulvar carcinoma, and the POP Quantification (POP-Q) scores showing Aa:+2, Ba:+2, C:0, D:0, Ap:+1, Bp:+1. The primary diagnoses is highly differentiated squamous cell carcinoma of the vulva, pelvic organ prolapse. The patient underwent extensive vulvotomy, vulvoplasty, partial urethrotomy, external urethroplasty, partial cervicectomy, anterior and posterior vaginal wall repair, sacrospinous ligament suspension, and laparoscopic bilateral inguinal lymph node dissection. The patient recovered well and was discharged from hospital. But unfortunately, the patient was followed-up via telephone and died 1 year after surgery.

Conclusions: Vulvar cancer and pelvic organ prolapse tend to occur in elderly individuals with other complications, such as heart disease, hypertension and diabetes. Risk during the perioperative period is very high; therefore, gynecologists should provide the most appropriate and comprehensive treatment plan to achieve a good prognosis.

\section{Background}

Pelvic organ prolapse is a common benign condition in women, defined as the descent of one or more of the following: anterior vaginal wall; posterior vaginal wall; uterus (cervix); and apex of the vagina (vaginal vault or cuff scar after hysterectomy). Vulvar cancer is uncommon, accounting for 2-5\% of gynecologic malignancies. Up to now, it is the first case of vulvar cancer complicated with POP. We will report about the treatment process. A 68-year-old, G3P3, female (BMI $\left.28 \mathrm{~kg} / \mathrm{m}^{2}\right)$ who suffered from the squamous cell carcinoma of the vulva associated with POP. The patient underwent extensive vulvotomy, vulvoplasty, partial urethrotomy, external urethroplasty, partial cervicectomy, anterior and posterior vaginal wall repair, sacrospinous ligament suspension, and laparoscopic bilateral inguinal lymph node dissection. The patient recovered well and was discharged from hospital. Vulvar cancer and pelvic organ prolapse tend to be elderly, and complications are more, such as heart disease, hypertension, diabetes. The risk of perioperative period is very high, and gynecologists need to provide the most appropriate treatment plan for patients with comprehensive factors, so as to bring good prognosis for patients. We hope this case can provide you with reference value. Informed consent was obtained from the patient to publish this case. 


\section{Case Presentation}

A 68-year-old woman (gravid 3, para 3) with a body mass index of $28 \mathrm{~kg} / \mathrm{m}^{2}$ ) and squamous cell carcinoma (SCC) of the vulva associated with pelvic organ prolapse (POP; in this case, prolapse of the anterior vaginal wall, posterior vaginal wall, and uterus) was admitted to our department in May 2018 for surgical treatment (Fig 1).

Her gynecological history included regular menstruation, three vaginal deliveries, and bilateral tubal ligation. Six months before admission, she had vulvar pruritis that was treated with antipruritic treatment. Three months before admission, she inadvertently found a mass in the vulva and no significant relief of pruritus. She did not experience overflow incontinence, dysuria, or urinary retention.

We discovered third-degree prolapse of the anterior vaginal wall and second-degree prolapse of the posterior vaginal wall and uterus according to Chinese standards. Her POP Quantification (POP-Q) scores were Aa:+2, Ba:+2, C:0, D:0, Ap:+1, Bp:+1. Pre-existing perineal and cervical lacerations were also observed. Cauliflower-like tissues of the clitoris and labia minora (size, $5^{\prime} 6^{\prime} 5 \mathrm{~cm}$ ) involving the urethral orifice were also observed. We did not find prolapse of the cystocele or rectocele. Vaginal and cervical biopsy results suggested highly differentiated SCC of the vulva and chronic cervicitis. Diagnostic ultrasound revealed no pathological changes in the uterus, fallopian tubes, and ovaries, and there was no renal urine retention. Of note, magnetic resonance imaging (MRI) showed thickening of the soft tissue in the vestibule and labia minora of the right part of the vulva, local nodular changes, and an unclear boundary of the external urethral orifice. Furthermore, MRI suggested lymph node enlargement in the bilateral external iliac vessels and inguinal region.

Because of the larger area of vulvar lesions and involvement of the urethral orifices, two chemotherapy treatments (bleomycin $15 \mathrm{mg} / 30 \mathrm{mg}$ on day $1 /$ day 2 and platinum $80 \mathrm{mg}$ on day 1 ) were administered before surgery. After routine preparation, we injected the cervix with nanocarbon developer. Next, we dissected the developed and undeveloped bilateral inguinal lymph nodes, during laparoscopy. Intraoperative freezing suggested left, but not right, inguinal lymph node metastasis.

After abdominal surgery, we treated the vulvar cancer with extensive vulvectomy and partial urethrotomy because the lesion invaded the urethra (Figs 2 and 3). Eventually, we treated POP with partial cervicectomy, anterior and posterior vaginal wall repair, and sacrospinous ligament suspension. After surgery, a cotton pad and bandage were applied to the vulva and groin. Of note, it was necessary to confirm that both dorsal foot arteries were normal.

The patient was discharged 5 days after surgery without lymphedema or wound infection. She underwent two courses of chemotherapy and 25 courses of radiotherapy after surgery at the local hospital. More than 5 months after surgery, a cauliflower-like pattern of tissue was seen at the urethral orifice and urethral biopsy results indicated SCC. Computed tomography (CT) performed at our hospital suggested that the dense shadow of soft tissue in the external orifice of the urethra was indistinguishable from the 
urethra and external orifice of the vagina. The patient was followed-up via telephone and died 1 year after surgery.

\section{Discussion And Conclusions}

Vulvar cancer is an uncommon disease, accounting for $\sim 5 \%$ of all gynecologic cancers, and SCC accounts for nearly $90 \%$ of vulvar cancer. [1,2] Vulvar cancer is most common in women $>60$ years; however, the incidence is increasing in women $<50$ years because of increasing prevalence of human papilloma virus (HPV).[3] A previous cohort study concluded that the incidence of vulvar cancer is significantly high for multiparous and postmenopausal women. [4]

Localized disease has been observed in $59 \%$ of patients, regional lymph node spread in $30 \%$, distant metastasis in $6 \%$, and unstaged disease in $5 \%$. The overall survival rate at 5 years is $71 \%$. Radical surgery, lymphadenectomy, and adjunctive radiation and chemotherapy are the main therapies for regionally advanced disease. Both surgical approaches (radical local excision or modified radical vulvectomy) involve resection of an approximately 1-2-cm margin of grossly normal tissue and deep fascia or a minimum tissue depth of $1 \mathrm{~cm}$.

Neoadjuvant chemoradiation is an effective modality for locally advanced vulvar cancer because it reduces the tumor size and allows for surgery of the lesion. Neoadjuvant chemotherapy has resulted in a considerable response rate of $>80 \%$ and a 5 -year survival rate of $92 \%$ after surgery. [5] Adjuvant chemotherapy has also increased the survival time and reduced mortality risk (44 vs 29.7 months; hazard ratio, 0.62; 95\% confidence interval, 0.48-0.79; $\mathrm{P}<.001)$.[6]

Larger lesions, especially those $>2 \mathrm{~cm}$, are associated with an increased risk of lymph node metastasis. Traditional radical inguinofemoral lymphadenectomy involves may complications, such as wound breakdown, infection, lymphocele, lymphedema, and wound infection; hence sentinel node evaluations have recently been advocated for early-stage cancers to reduce these complications and short-term and long-term morbidity rates. A recent meta-analysis indicated that the overall sensitivity for the identification of sentinel node metastasis using complete inguinofemoral node dissection is $92 \%$. Candidates for sentinel lymph node biopsy should have clinically/radiologically negative groin nodes, unifocal primary tumors $<4 \mathrm{~cm}$, and no history of previous vulvar surgery.

Recurrence risk is associated with the depth of invasion, presence of carcinoma at the surgical margin, and histologic grade. Tobacco use and HPV infection increase the risk of vulvar cancer, and the persistence of these factors may increase the risk of recurrence. A meta-analysis indicated that the HPV status may have an important role in predicting the prognosis of vulvar cancer. Women with HPV-positive vulvar cancer might have relatively better survival that those with HPV-negative vulvar cancer. [7]

Because of the high recurrence rates of $12-39.5 \%$ for all vulvar SCC cases, routine surveillance is recommended after primary treatment. Most recurrences occur within the first 2 years after treatment (32.7\% of patients with node-positive cancer and $5.1 \%$ of women with negative nodes). The average time 
at which patients with nodal metastatic disease experience recurrence in the groin is 10.5 months. Therefore, the Society of Gynecologic Oncologists and the National Comprehensive Cancer Network recommend surveillance with history and detailed physical examination every 3 months for the first 2 years after treatment.

Based on a retrospective review of 586 patients enrolled in Gynecologic Oncology Group trials in 1984, independent predictors of survival includ the presence of involved lymph nodes, number of involved lymph nodes, and primary tumor size. Lymph node metastasis is considered the most important prognostic factor and determinant of treatment in vulvar cancer, and extracapsular extension has been linked to poorer prognoses. Additional factors predictive of recurrence and/or survival include depth of invasion, tumor thickness, and lymphovascular space invasion.

Current surgical approaches involve primary tumor resection and lymph node evaluation based on the characteristics of the individual patient. The risk-to benefit ratio and morbidity associated with each approach must be evaluated and individualized for each patient as well. Further emphasizing the importance of adequate inguinofemoral lymph node evaluation and treatment at the time of initial presentation, it has been widely reported that subsequent relapse in the groin is rarely amenable to successful secondary treatment. Radical surgery of the vulva and groin lymph nodes usually requires prolonged recovery.

POP is defined as the descent of one or more of the following: anterior vaginal wall; posterior vaginal wall; uterus (cervix); and apex of the vagina (vaginal vault or cuff scar after hysterectomy). It can significantly affect the quality of life in women of all ages. An estimated $3 \%$ and $4.1 \%$ of women aged 60 79 and $>80$ years, respectively, are affected by POP [8,9]; however, the true prevalence is unknown because many women are unaware of their prolapse or do not seek help.

Prolapse is typically multifactorial, but is primarily associated with pregnancy and vaginal delivery, which lead to direct pelvic floor muscle and connective tissue injuries. Based on vaginal examination, POP occurs in ${ }^{3} 50 \%$ of parous women. Hysterectomy, pelvic surgery, and conditions associated with sustained episodes of increased intra-abdominal pressure, including obesity, chronic cough, constipation, and repeated heavy lifting, also contribute to prolapse.

Most POP patients are asymptomatic. However, symptoms become bothersome as the bulge protrudes past the vaginal opening. POP is dynamic. Its symptoms and examination results may vary temporally, depending on the level of activity and fullness of the bladder and rectum. Prolapse often coexists with other pelvic floor disorders. Forty percent of prolapse patients report stress urinary incontinence, $37 \%$ overactive bladder, and $50 \%$ fecal incontinence. POP may negatively affect sexual activity, body image, and quality of life. The POP-Q system has been employed in clinical practice and research.

POP's initial diagnostic evaluation includes the medical history and systematic pelvic examination including an assessment to determine urinary incontinence, bladder outlet obstruction, and fecal incontinence. Other tests include measurements of urethral activity, evaluation of bladder function, and 
evaluation of urine flow mechanics. For cases of recurrent or unusual prolapse (e.g., large perineal bulge), defecation proctography or dynamic pelvic MRI may be useful.

Women with POP may elect observation (expectant management), pelvic floor muscle training, pessary use, or surgery as treatment. Lifestyle modifications, such as smoking cessation and avoiding heavy lifting and constipation, may reduce symptoms. Pessaries are an option for all stages of prolapse and may prevent prolapse progression and avert of delay the need for surgery.

Obliterative and reconstructive surgeries for POP are available and include hysterectomy and uterine conservation (hysteropexy). The decision to undergo surgery must include a discussion of the patient's goals and expectations based on cultural views such as body image and desire for future sexual function, including vaginal intercourse. Vaginal obliteration (colpocleisis) has the highest cure rate and lowest morbidity of any surgical intervention; it is an excellent option for women who do not desire any future vaginal intercourse. Surgical repair of POP is challenging and fraught with a high reoperation rate of ${ }^{3} 29 \%$ [10]. The mean time to first reoperation for recurrent prolapse after primary surgical correction is 3-4 years. The aims of prolapse repair are symptomatic relief, anatomic and functional restoration of the pelvic structures, recurrence prevention, and concomitant intrapelvie defect repair.

Vulvar cancer and POP are common in elderly women and are accompanied by complications, such as hypertension, diabetes, and venous thrombosis. Vulvar carcinoma with POP is rare; therefore, its treatment is complicated. Furthermore, the treatment of vulvar cancer remains challenging for gynecologic oncologists. Therefore, a common and standard definition is critical to maximize oncological results and minimize complications after treatment. Treatment must be tailored to each individual according to their clinical and biological features. Because most patients are elderly, a suitable surgical method based on a variety of factors to reduce the burden of surgery and facilitate rapid recovery after surgery must be selected. We hope that the details of this case will be helpful for the diagnosis and treatment of similar cases in the future.

\section{Declarations}

Ethics approval and consent to participate: Ethics approval on record for this study was given by the ethics committee of West China Second Hospital of Sichuan University.

Consent for publication: Written informed consent was obtained from the patient's families for publication of this case report and any accompanying images.

Availability of data and materials: Not applicable.

Competing interests: Not applicable.

Funding: Not applicable.

\section{Authors' contributions:}


Qin Tang: Software, Writing-original draft.

Ping Wang: Conceptulization, Supervision, Writing-review \& editing.

Acknowledgements: Not applicable.

\section{References}

1. Rogers LJ, Cuello MA. Cancer of the vulva. International Journal of Gynecology Obstetrics $2018 ; 143$ suppl 2:4-13. https://doi.org/ 10.1002/ijgo.12609.

2. Weinberg D, Gomez-Martinez RA. Vulvar Cancer. Obstet Gynecol Clin North Am. 2019;46(1):125-35. https://doi.org/10.1016/j.ogc.2018.09.008.

3. Viswanathan C, Kirschner K, Truong M, Balachandran A, Devine C, Bhosale P. Multimodality Imaging of Vulvar Cancer: Staging, Therapeutic Response, and Complications. Am J Roentgenol. 2013;200(6):1387-400. https://doi.org/10.2214/AJR.12.9714.

4. Singh N, Negi N, Srivastava K, Agarwal G. A cohort study of vulvar cancer over a period of 10 years and review of literature. Indian J Cancer 2016; 53(3): 412-5. https://doi.org/ 10.4103/0019509X.200656.

5. Aragona AM, Cuneo N, Soderini AH, Alcoba E, Greco A, Reyes C, et al. Tailoring the Treatment of Locally Advanced Squamous Cell Carcinoma of the Vulva: Neoadjuvant Chemotherapy Followed by Radical Surgery. International Journal of Gynecologic Cancer 2012; 22(7): 1258-63. https://doi.org/ 10.1097/IGC.0b013e318263ef55.

6. Koh WJ, Greer BE, Abu-Rustum NR, Campos SM, Cho KR, Chon HS, et al. Vulvar Cancer, Version 1.2017, NCCN Clinical Practice Guidelines in Oncology. J Natl Compr Canc Netw. 2017;15(1):92-120. https://doi.org/10.6004/jnccn.2017.0008.

7. Zhang J, Zhang Y, Zhang Z. Prevalence of human papillomavirus and its prognostic value in vulvar cancer: A systematic review and meta-analysis. PLoS ONE. 2018;13(9):e0204162. https://doi.org/1371/journal.pone.0204162.

8. Cheryl B, Iglesia MAKR. Pelvic Organ Prolapse. Female Pelvic Medicine Reconstructive Surgery. 2017;23(6):353-64. https://doi.org/10.1097/SPV.0000000000000498.

9. Anderson KM, Davis K, Flynn BJ. Urinary Incontinence and Pelvic Organ Prolapse. Med Clin North Am. 2015;99(2):405-16. https://doi.org/10.1016/j.mcna.2014.11.011.

10. Krlin RM, Soules KA, Winters JC. Surgical repair of pelvic organ prolapse in elderly patients. Curr Opin Urol. 2016;26(2):193-200. https://doi.org/10.1097/MOU.0000000000000260.

\section{Figures}




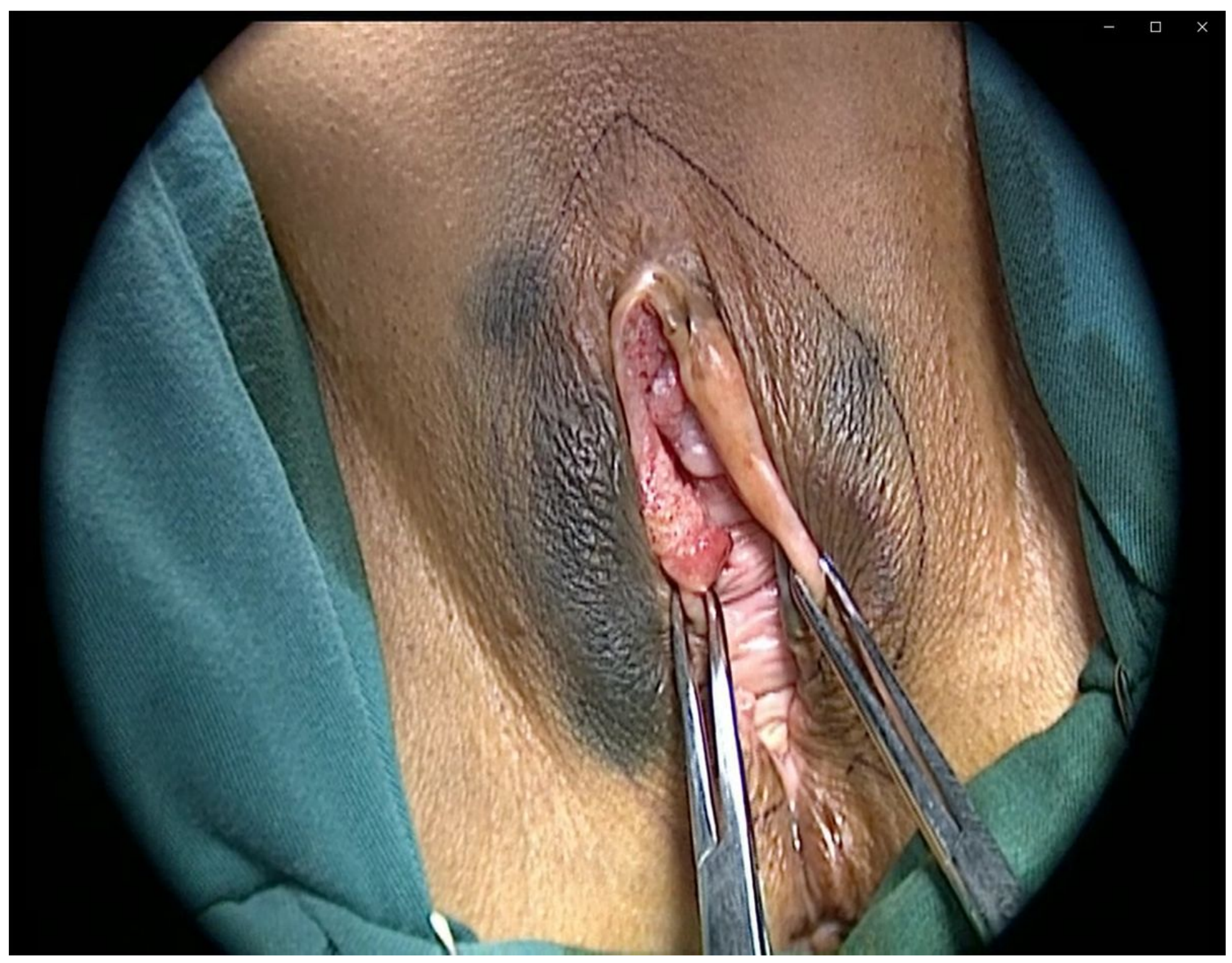

Figure 1

The patient with vulvar cancer and pelvic organ prolapse. 


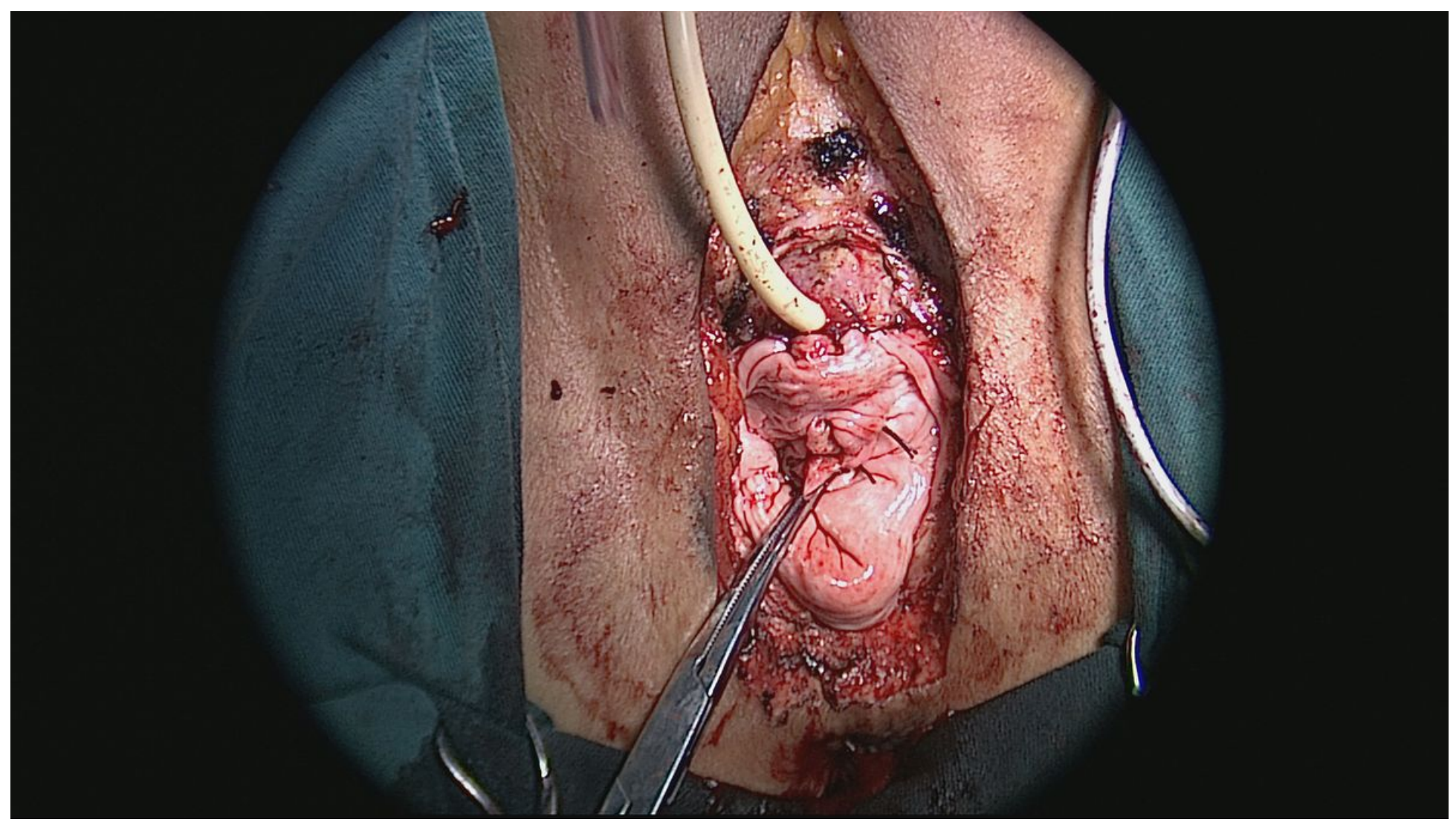

Figure 2

The extent of vulva removal. 


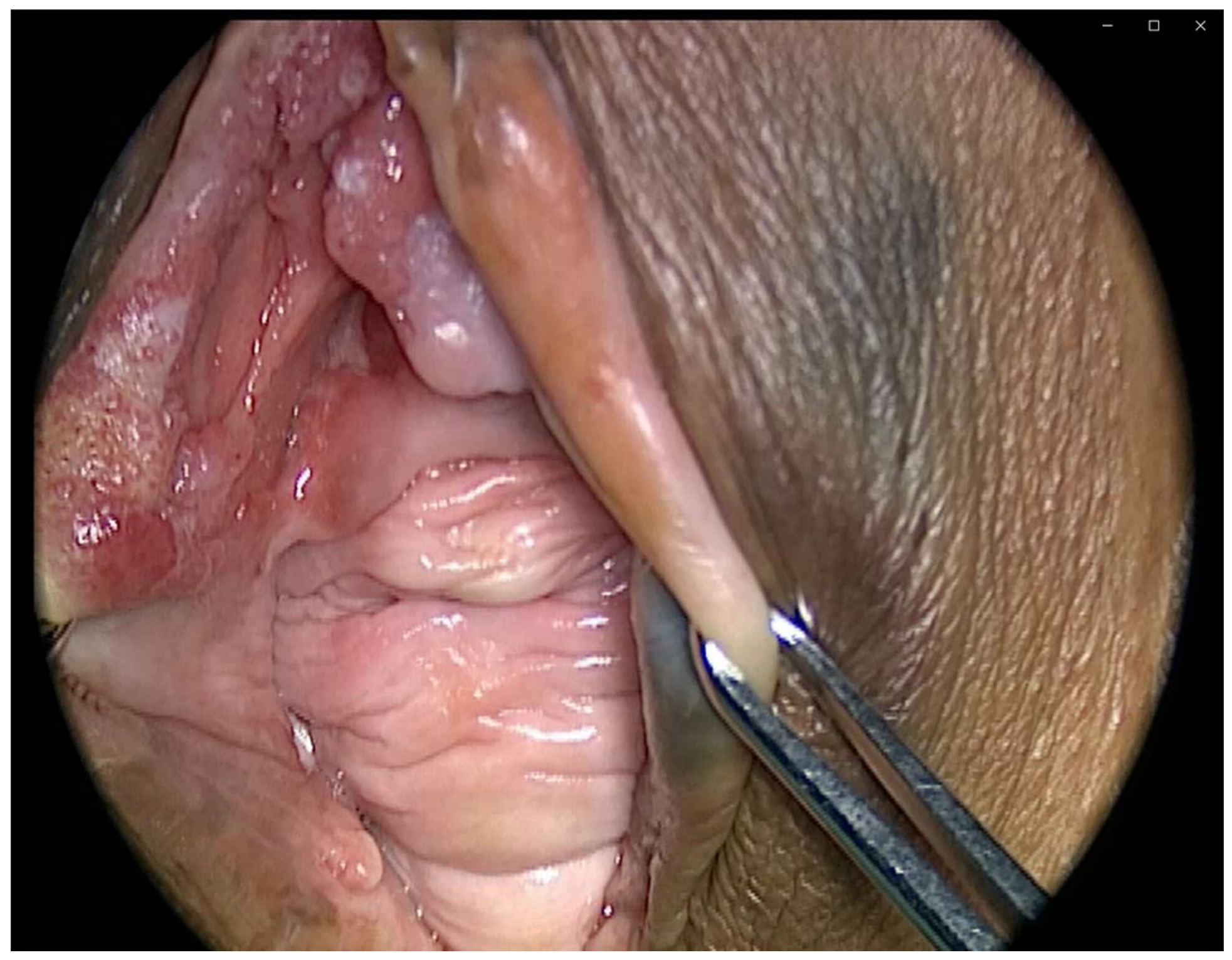

Figure 3

Changes after vulvar resection. 